\title{
Loading and unloading of sedimentary basins: The effect of rheological hysteresis
}

\author{
A. C. Fowler \\ Mathematical Institute, University of Oxford, Oxford, UK
}

\author{
Xin-She Yang \\ Department of Applied Mathematics, University of Leeds, Leeds, UK
}

Received 23 March 2000; revised 22 September 2001; accepted 27 September 2001; published 10 April 2002

[1] We present a model for the study of the effect of rheological hysteresis on compaction in sedimentary basins, when surface loading and unloading occurs. When compaction is slow (sedimentation rate is large and/or permeability is small), the hysteresis has little effect on the basal compaction layer, but in the more realistic case where compaction is fast (i.e., sedimentation is slow and/or permeability is large), surface unloading leads to downward propagation of a decompaction front, across which the vertical porosity gradient jumps, while subsequent surface reloading leads to downward propagation of a discontinuity in porosity, despite the fact that the porosity is governed by a diffusive equation of Richards type. INDEX TERMS: 3022 Marine Geology and Geophysics: Marine sediments-processes and transport; 3210 Mathematical Geophysics: Modeling; 8105 Tectonophysics: Continental margins and sedimentary basins; KEYWORDS: Compaction, sedimentary basins, hysteresis

\section{Introduction}

[2] The compaction of sedimentary basins is a problem of particular interest in the oil industry because of the accumulation of hydrocarbons within the sediments. As the sediments compact, they expel pore water, and if the compaction is sufficiently rapid, or the sediment permeability is sufficiently low, then excess pore pressures (above hydrostatic) may occur, and this is of serious concern in drilling operations.

[3] In the past, most studies of compaction have focused on the accumulation of sediments, but in reality, tectonically induced alterations in topography over geologic time lead to a succession of periods of sediment loading and unloading. For example, the North Sea is a patchwork of basins which appear to have undergone subsidence since the mid-Cretaceous (100 Ma), this being preceded by uplift and erosion [Sclater and Christie, 1980] and an associated jump in the age of the rocks at depth. It has been suggested that massive erosion also took place at the Cretaceous-Tertiary boundary [Lewis et al., 1992]; tertiary uplift due to magmatic underplating in the North Sea about 65 Myr ago [Clift and Turner, 1998], followed by the opening of the Atlantic Ocean, caused a period of erosion, since when further sediment accumulation and subsidence has occurred. Hillis [1995] estimates that about a kilometer of uplift and exhumation took place at rates of $100 \mathrm{~m} \mathrm{Myr}^{-1}$, while overall burial rates of the order of $30 \mathrm{~m} \mathrm{Myr}^{-1}$ have been inferred [Allen and Allen, 1990]. This is consistent with stratigraphy of well sections, which indicates various missing rock ages in various parts of the basin complex [Shannon and Naylor, 1989, p. 83]. A further example (which we discuss in more detail below) is in the North China Basin [Wang, 1992], where marine sedimentation appears to have been followed by uplift, erosion, and subsequent subaerial deposition. Cercone [1984] has suggested that erosion occurred in the Michigan Basin to remove up to $1000 \mathrm{~m}$ of Carboniferous strata, and Gerlach and Cercone [1993] have proposed that similar erosion occurred in the Appalachian Basin.

Copyright 2002 by the American Geophysical Union. 0148-0227/02/2001JB000389\$09.00
[4] There are two particular possible effects of periods of alternating erosion and deposition. One is simply that a change in depositional environment may lead to unconformities, and these are commonly seen. The second, and the purpose of our study, is that jumps in porosity may occur. Sclater and Christie [1980] show an example from the North Sea (their Figure A2, redrawn here in Figure 1), in which there is a distinct decrease of porosity at a depth of about $3000 \mathrm{~km}$, where the sediments change rapidly from Paleocene age to Jurassic.

[5] Of course, a change of sediment type can in itself cause changes in porosity, and furthermore, well log data are often very noisy, so that hysteresis-induced porosity jumps may often be masked. However, clean examples in data do exist, and in the conclusions, we will show a very good example of this jump in a well log from the North China Basin. Hysteresis is also sometimes assumed in interpreting well log data; for example, Issler [1992] uses the concept of surface porosity deficit in unloading sediments to estimate the amount of surface erosion in the Beaufort-MacKenzie basin, Canada, and similar inferences have been made by Magara [1976b]. On the assumption that porosity jumps can be due to rheological hysteresis, in this paper we wish to generalize the use of compaction models in order to explain how discontinuous porosity profiles may arise and be maintained.

[6] Early studies of compaction were carried out by Athy [1930] and Hedberg [1936], and more recent work includes that of Gibson [1958], Smith [1971], Rieke and Chilingarian [1974], Wangen [1992], and Fowler and Yang [1998]. Some of this (and other) work was reviewed by Audet and Fowler [1992], whose formalism we follow here. Such previous studies of compaction assume a rheological behavior which is reversible: for example, purely (nonlinear) elastic behavior. However, it is well known in the analogous field of soil consolidation that the rheological behavior is hysteretic and irreversible [Wood, 1990], and experimental results on sediment deformation to pressures of $70 \mathrm{MPa}$ (corresponding to depths of 3-4 km) are consistent with this [Jones, 1994]. The purpose of the present paper is to devise a suitable model to describe such irreversible behavior and to exhibit some of its novel effects. Although Athy [1930] described compaction in 


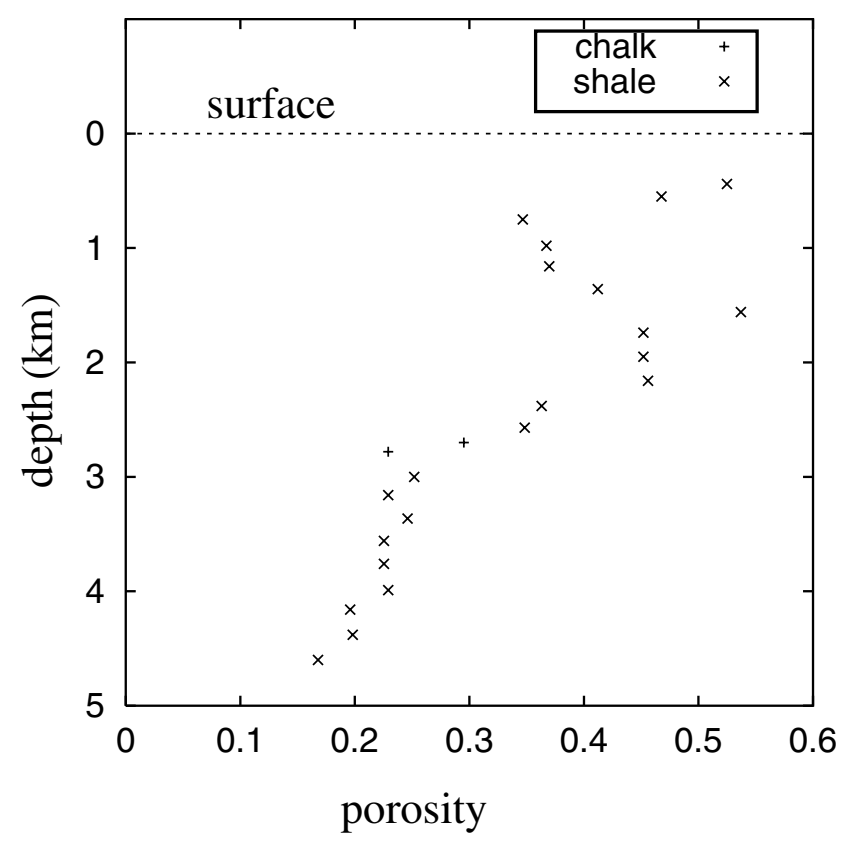

Figure 1. Porosity versus depth for Amoco well 2/11-1 in the North Sea Basin, redrawn from Sclater and Christie [1980]. There is a clearly identifiable drop in porosity at a depth of $2500 \mathrm{~m}$, corresponding to a mid-Cretaceous erosional unconformity. The rock at this depth is chalk, with shale lying above and below. The effect is clouded by the overpressuring in the Tertiary shales at $1.5-2 \mathrm{~km}$, associated with the raised porosity levels.

shales using soil mechanics concepts (as did Jones [1994]), one might question whether the analogy can be extended to sediment depths of thousands of meters. The compaction which occurs in soils occurs through grain rearrangement, and this ought to describe sediment compaction also, at least at shallow depths. At deeper levels, pressure solution of grains may become important, and then the appropriate compactive rheology will be a viscous one [Birchwood and Turcotte, 1994; Fowler and Yang, 1999]. Some inference of the appropriateness of an irreversible elastic behavior at the geologic scale lies in the observation of subsidence due to groundwater removal, for example, in Venice [Lewis and Schrefler, 1987] and also in the brittle nature of exposed surface rocks, typical of overconsolidated sediments [Jones, 1994].

[7] We thus suppose that compaction, at least in relatively shallow sediments (before pressure solution or cementation occur), is analogous to the process of soil consolidation. The sediments act as a compressible porous matrix, so that mass conservation of pore fluid together with Darcy's law leads to an equation of the general type $\partial \phi / \partial t+\nabla \cdot \mathbf{q}=0, \mathbf{q} \propto-\nabla p$, where $\phi$ is porosity, $\mathbf{q}$ is fluid flux, and $p$ is fluid pressure. This model must be supplemented by a constitutive law relating pore pressure $p$ to porosity $\phi$, and in soil mechanics this takes (most simply) the form of a nonlinear elastic relationship between the effective pressure $p_{e}=P-p$, where $P$ is the overburden pressure, and $\phi$, whose graph is called the normal consolidation line. It is roughly given by a straight line when plotted using coordinates of void fraction $\phi /(1-\phi)$ and $\ln p_{e}$ (see Figure 2), and is usually written in the form [Bowles, 1984]

$$
e=e_{0}-C_{c} \log _{10}\left(p_{e} / p_{e}^{0}\right)
$$

where $e=\phi /(1-\phi) ; C_{c}$ is the compression index. If the natural logarithm is used, then the value of $C_{c}$ needs to be multiplied by $2.3(=\ln 10)$. The linear relation between $e$ and $\ln p_{e}$ becomes invalid at very low or very high $p_{e}$.

[8] The main complication concerns the assumed rheology. Even for soils, the relationship $\phi=\phi\left(p_{e}\right)$ is irreversible, exhibiting hysteresis, and incorporation of this into basin loading/unloading histories complicates the model and analysis. The one-dimensional isotropic consolidation test of soil accompanied by unloading/reloading sequences [Das, 1983; Burland, 1990] clearly shows that the soil behavior is path-dependent and nonlinear as shown in Figure 2. The behavior during unloading and reloading is essentially elastic with a small amount of hysteresis. The nonlinear behavior inherent in Figure 2 has been described

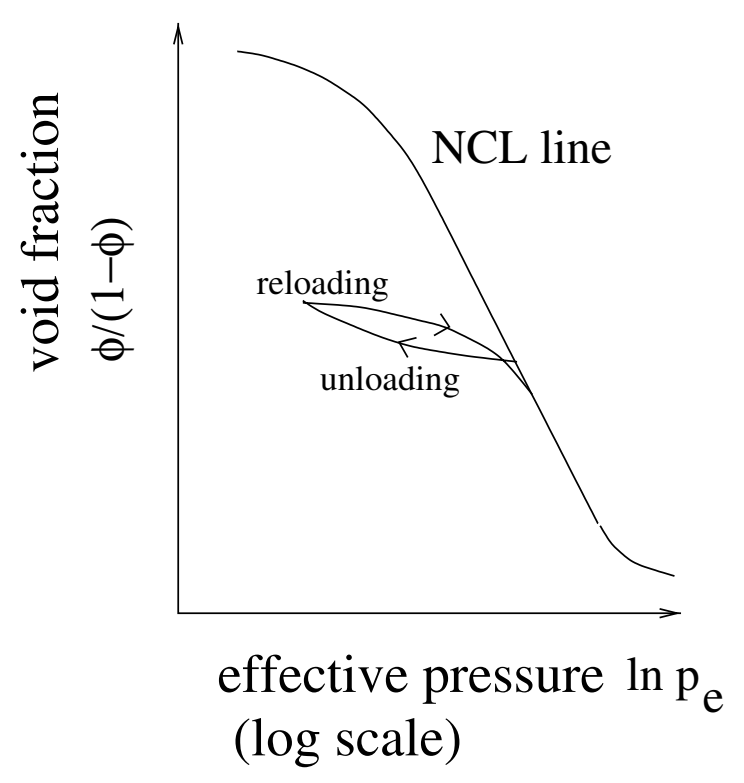

(a)

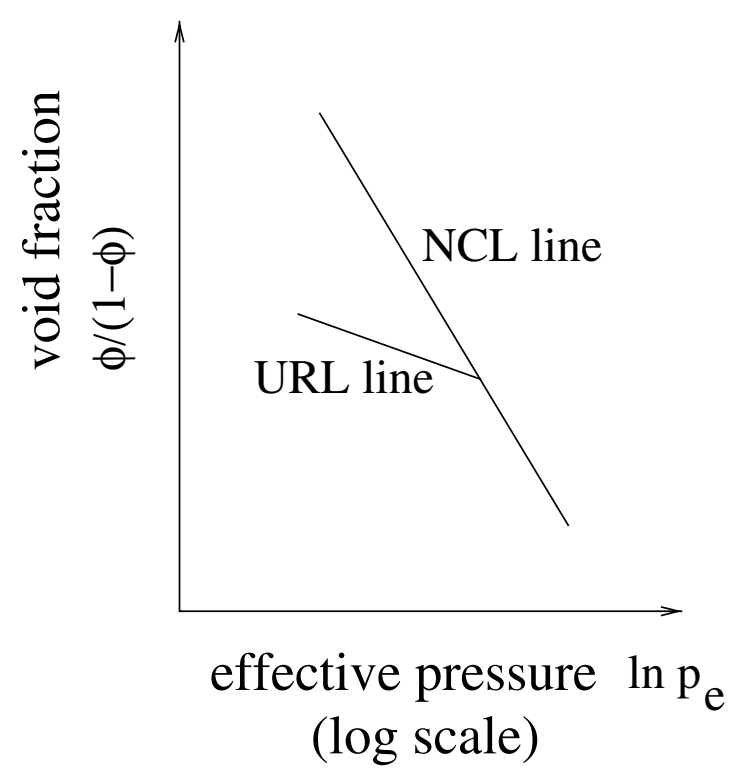

(b)

Figure 2. Nonlinear behavior of soil consolidation: (a) typical experimental result for the normal consolidation line (NCL) and the hysteretic effect of unloading and reloading. (b) Corresponding idealized model of loading/unloading. URL is the unloading and reloading line. 
Table 1. Variables and Parameters of the Model

\begin{tabular}{|c|c|c|c|}
\hline Symbol & Meaning & Definition & Typical Value \\
\hline$\phi$ & porosity & (2) & $<1$ \\
\hline$u^{l}$ & liquid velocity & (2) & \\
\hline$u^{s}$ & solid velocity & (3) & \\
\hline$k$ & permeability & (4) & $\lesssim \mathrm{k}_{0}$ \\
\hline$\mu$ & liquid viscosity & (4) & $10^{-3} \mathrm{~Pa} \mathrm{~s}$ \\
\hline$p$ & pore pressure & (4) & \\
\hline$\rho_{l}$ & liquid density & (4) & $10^{3} \mathrm{~kg} \mathrm{~m}^{-3}$ \\
\hline$g$ & gravity & (4) & $9.8 \mathrm{~m} \mathrm{~s}^{-2}$ \\
\hline$M$ & elastic constant & (5) & $O(1)$ \\
\hline$p_{e}$ & effective pressure & (5) & \\
\hline$\rho_{s}$ & sediment density & (5) & $2.6 \times 10^{3} \mathrm{~kg} \mathrm{~m}^{-3}$ \\
\hline$G$ & shear modulus & (6) & \\
\hline$\Delta$ & dilation & (6) & \\
\hline$\tilde{p}_{e}$ & scaled $p_{e}$ & (12) & \\
\hline$\tilde{k}$ & scaled $k$ & (11) & \\
\hline$k_{0}$ & permeability scale & (10) & $\begin{array}{l}\sim 10^{-13} \mathrm{~m}^{2} \text { (sandstone) } \\
\sim 10^{-16} \mathrm{~m}^{2} \text { (shale) }\end{array}$ \\
\hline$\lambda$ & compaction parameter & (10) & $\begin{array}{l}10^{2} \text { (shale) } \\
10^{5} \text { (sandstone) }\end{array}$ \\
\hline$v_{s}$ & $\begin{array}{l}\text { sedimentation rate } \\
\text { rate scale }\end{array}$ & (10) & $100 \mathrm{~m} \mathrm{Myr}^{-1}$ \\
\hline$\phi_{0}$ & surface porosity & (11) & 0.5 \\
\hline$\gamma$ & URL parameter & (13) & 0.2 \\
\hline$d$ & depth scale & after (6) & $2 \mathrm{~km}$ \\
\hline$v$ & sedimentation rate & e.g., in (16) & $v_{S} \sim 100 \mathrm{~m} \mathrm{Myr}^{-1}$ \\
\hline$\tilde{p}_{e}{ }^{*}$ & $\tilde{p}_{e}$ at $\mathrm{URL} / \mathrm{NCL}$ join & (18) & \\
\hline$t$ & time & & $\sim d / v_{S} \sim 20 \mathrm{Ma}$ \\
\hline$z$ & height above basement & & $\sim d$ \\
\hline$h(t)$ & sediment thickness & & $1-10 \mathrm{~km}$ \\
\hline$\Pi$ & pressure scale & (7) & \\
\hline
\end{tabular}

by the Cam-Clay model [Schofield and Wroth, 1986; Roscoe and Burland, 1968] and the more modern cap model [Chen and Mizuno, 1990], in which only volumetric strain is assumed to be partially recoverable. In our description, we will suppose that the consolidation curves in Figure 2a are idealized as those shown in Figure $2 \mathrm{~b}$. In particular, the unloading and reloading curves are supposed to coincide as the unloading/reloading line (URL).

\section{Mathematical Model}

[9] The basic model of compaction describes the one-dimensional flow of both solid and liquid phases and is based on the framework developed by Audet and Fowler [1992] and Fowler and Yang [1998]. Table 1 defines the variables and parameters of the model.

[10] For a one-dimensional basin $0<z<h(t)$, where $h(t)$ is the ocean floor and $z=0$ is the basement rock, the governing model equations for one-dimensional compaction can be written as

Mass conservation

$$
\begin{gathered}
\frac{\partial \phi}{\partial t}+\frac{\partial}{\partial z}\left(\phi u^{l}\right)=0 \\
-\frac{\partial \phi}{\partial t}+\frac{\partial}{\partial z}\left[(1-\phi) u^{s}\right]=0 ;
\end{gathered}
$$

Darcy's law

$$
\phi\left(u^{l}-u^{s}\right)=-\frac{k}{\mu}\left(\frac{\partial p}{\partial z}+\rho_{l} g\right)
$$

Force balance

$$
\frac{\partial p}{\partial z}\left(-M p_{e}-p\right)-\left[\rho_{s}(1-\phi)+\rho_{l} \phi\right] g=0 .
$$

In (2)-(5), $\phi$ is the porosity; $u^{l}$ and $u^{s}$ are the velocities of fluid and solid matrix; $\rho_{l}$ and $\rho_{s}$ are the densities of the fluid and of the solid matrix, respectively, which are taken as constant, i.e., the fluid and solid phases are separately incompressible; $p_{e}$ and $p$ are effective pressure and pore water pressure, respectively; $k$ is the matrix permeability; $\mu$ is the liquid viscosity; $g$ is the gravitational acceleration; and $M$ is an elastic constant, which is defined by

$$
M=1+\frac{4 G}{3\left[-\frac{d p_{e}}{d \phi}\left(\frac{1-\phi}{1-\Delta}\right)\right]},
$$

where $\Delta$ is the dilation $\partial U / \partial z, U$ being the vertical (matrix) displacement, and $G$ is the shear modulus [Fowler and Yang, 1998]. Although the motion is assumed one-dimensional, the stress field is not, since hoop stresses are necessary in a uniaxial strain field. These have been incorporated here in the definition of $M$. This model must be supplemented by a constitutive law for $p_{e}(\phi)$ with (if we include the effects of hysteresis) separate branches for normal consolidation line (NCL) and URL, discussed further below.

[11] Suppose that the effective pressure is written in the form

$$
p_{e}=\Pi \tilde{p}_{e}(\phi)
$$

where $\Pi$ is the pressure scale (so that the dimensionless effective pressure $\tilde{p}_{e}$ is $\left.O(1)\right)$. The value of $\Pi$ distinguishes between sediment types. We then choose the depth scale to be

$$
d=\frac{M \Pi}{\left(\rho_{s}-\rho_{l}\right) g} \text {. }
$$

Suppose that a typical value of the sedimentation rate is denoted $v_{S}$, then we use this as our velocity scale, and $d / v_{S}$ as the timescale. In addition, the permeability is scaled with a reference value $k_{0}$ (when $\left.p_{e}=0\right)$. Next we write (2)-(5) in dimensionless terms using these scales.

[12] To reduce the model, we then integrate the sum of the mass conservation equations with the condition of an impermeable basement at $z=0$; this allows us to write $\phi\left(u^{l}-u^{s}\right)$ in terms of $u^{s}$, and thus Darcy's law (4) relates $u^{s}$ to $\partial p / \partial z$ and thence, via (5), to $\partial p_{e} / \partial z$. Finally, we use the assumption that $p_{e}$ is a function of $\phi$ and substitute the expression for $u^{s}$ back into (3), which thus becomes a single nonlinear diffusion equation for $\phi$ :

$$
\frac{\partial \phi}{\partial t}=\lambda \frac{\partial}{\partial z}\left\{\tilde{k}(1-\phi)\left[-\frac{\partial p_{e}}{\partial z}-(1-\phi)\right]\right\},
$$

where the parameter $\lambda$ is given by

$$
\lambda=\frac{v_{H}}{v_{s}}=\frac{k_{0}\left(\rho_{s}-\rho_{l}\right) g}{\mu v_{s}}
$$

it is the ratio of the deposited sediment hydraulic conductivity $v_{H}=$ $k_{0}\left(\rho_{s}-\rho_{l}\right) g / \mu$ to the deposition rate $v_{S}$ (measured as a rate of thickness change). For later purposes, it is useful to get some idea of typical values of $\lambda$. Typical sedimentation rates are of the order of $100 \mathrm{~m} \mathrm{Myr}^{-1}$; for example, in the Gulf Coast $v_{S} \lesssim 160 \mathrm{~m} \mathrm{Myr}^{-1}$ [Magara, 1976a], while in the North Sea one can infer rates $v_{S} \lesssim 30$ $\mathrm{m} \mathrm{Myr}^{-1}$ [Sclater and Christie, 1980], although Tertiary exhumation may imply higher values. Magara [1976a] indicates permeabilities $\gtrsim 50$ mdarcy for sandstones, and $\lesssim 0.05$ mdarcy for shales. These correspond to values $\left(1\right.$ mdarcy $\left.=0.987 \times 10^{-15} \mathrm{~m}^{2}\right)$ of $\gtrsim 0.5 \times 10^{-13} \mathrm{~m}^{2}$ for sandstone and $\$ 0.5 \times 10^{-16} \mathrm{~m}^{2}$ for shale. The range given by Freeze and Cherry [1979] is $10^{-2}-10^{2}$ mdarcy for sandstone and $10^{-5}-10^{-1}$ mdarcy for shale, and these ranges are consistent bearing in mind the variation in permeability 


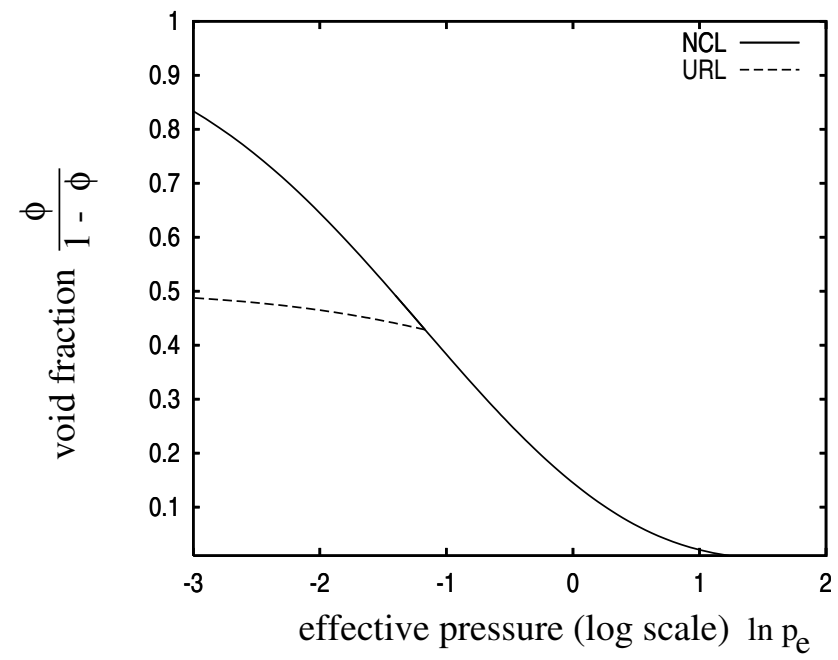

Figure 3. Void fraction $\phi /(1-\phi)$ versus $\ln p_{e}$ for the Smith relation in equation (12), with $\phi_{0}=0.5$. Also shown is the URL given by equation (13), when $\phi_{0}^{*}=0.3$ and $\gamma=0.2$. (The position of the URL depends on the maximum effective pressure reached in the past, and one illustrative example is shown here.)

with porosity. As estimates for $k_{0}$, we therefore choose $k_{0}=10^{-13}$ $\mathrm{m}^{2}$ (100 mdarcy) for sandstone and $k_{0}=10^{-16} \mathrm{~m}^{2}$ ( 0.1 mdarcy) for clay. We use values $\rho_{s}-\rho_{l}=1.6 \times 10^{3} \mathrm{~kg} \mathrm{~m}^{-3}, g=9.8 \mathrm{~m} \mathrm{~s}^{-2}$, and $\mu=1.57 \times 10^{-3} \mathrm{~Pa} s$ (the value a few degrees above freezing; it decreases by a factor of 6 at $100^{\circ} \mathrm{C}$ at atmospheric pressure); then the deposited hydraulic conductivity $v_{H}$ lies in the range $10^{-6} \mathrm{~m} \mathrm{~s}^{-1}$ (sandstone) to $10^{-9} \mathrm{~m} \mathrm{~s}^{-1}$ (shale), i.e., $3 \times 10^{7}$ to $3 \times 10^{4} \mathrm{~m} \mathrm{Myr}^{-1}$, and we see that for typical sedimentation rates $v_{S} \sim 100 \mathrm{~m} \mathrm{Myr}^{-1}$, the value of $\lambda$ lies in the approximate range $10^{2}-10^{5}$. Generally, therefore, $\lambda \gg 1$, and we will be largely concerned with this limit.

[13] To complete the model, we choose the (dimensionless) permeability function $\tilde{k}$ to be

$$
\tilde{k}=\left(\phi / \phi_{0}\right)^{m}
$$

A typical value of $m$ is 8 [Smith, 1971] and is distinguished from the normal values of 2-3 associated with the Carman-Kozeny law for granular materials by the enhanced chemical effects exhibited by clay minerals. We suppose that the equivalent of the normal consolidation line is [Fowler and Yang, 1998]

$$
\tilde{p}_{e}=\ln \left(\phi_{0} / \phi\right)-\left(\phi_{0} / \phi\right)
$$

different choices of these functions can be made if required.

[14] The choice of (12) is not based on actual compression data but rather is a choice motivated by the observation that many normally pressured well logs give an exponential decrease of porosity with depth [Athy, 1930; Hedberg, 1936; Dickinson, 1953; Sclater and Christie, 1980], and (12) enables this observation to be reproduced by the theory.

[15] As described previously, we would expect that elastic consolidation of sediments (which essentially arises through particle rearrangment, not creep or compression) should behave similarly to the well-established consolidation of soils. However, (12) appears very different to the soil consolidation law (1). In fact, this is not the case, as can be seen from Figure 3, which plots void ratio versus $\ln p_{e}$ for equation (12).

[16] The NCL equation (12) applies when $\phi$ is decreasing; as we have discussed, it is not based on direct experiment but on a choice which is consistent with observations of exponential decrease of porosity in normally pressured sediments with depth and is also, in fact, consistent (Figure 3) with the normal consolidation line (equation (1)). Since in unloading soils the void ratio follows an expression similar to (1) but with a lower coefficient $\left(C_{s}\right.$, the swelling index), it is natural here to propose a relationship on the URL which is analogous to (12), and we thus choose

$$
\tilde{p}_{e}=\left[1-(1-\gamma) \phi_{0}^{*}\right] \ln \left[\frac{\phi_{0}^{*} \gamma}{\phi-(1-\gamma) \phi_{0}^{*}}\right]-\left(\phi_{0}^{*}-\phi\right)+\tilde{p}_{e}^{*}
$$

with

$$
\begin{gathered}
\phi_{0}^{*}(z, t)=\min _{\tau<t} \phi(z, \tau), \quad \tilde{p}_{e}^{*}(z, t)=\max _{\tau<t} \tilde{p}_{e}(z, \tau), \\
\tilde{p}_{e}^{*}=\ln \left(\phi_{0} / \phi_{0}^{*}\right)-\left(\phi_{0} / \phi_{0}^{*}\right),
\end{gathered}
$$

and this applies if $\tilde{p}_{e} \leq \tilde{p}_{e}^{*}$ and $\phi \geq \phi_{0}^{*}$. In (13), $\gamma$ is the slope ratio of the URL line to the NCL line at their join. Just as we found that the functionally distinct formulae (12) and (1) actually have similar graphs, so also the unloading curve (13) resembles its soil consolidation counterpart (compare Figure 3 with Figure 2). According to Das [1983], the ratio for soils is $\gamma \approx 0.1 \sim 0.2$. Clearly, (13) degenerates into (12) when $\gamma=1$, which corresponds to the case that the URL line is identical to the NCL line. In this case, unloading and reloading are reversible processes.

[17] The boundary conditions that we apply to the model represent the condition of an impermeable basement at $z=0$, equality of pore pressure to ocean or atmosphere pressure at the sediment surface (depending on whether loading or unloading is occurring), and a kinematic condition at the sediment surface which determines the evolution of the sediment surface through the accumulation of new sediment. The second of these conditions deserves some consideration [Haxby and Turcotte, 1976]. One might suppose that in a case of rapid tectonic uplift the situation should be modeled by imposing a sudden reduction in overburden pressure. Even if the surface pore pressure adjusts instantaneously, there would be a zone of negative effective pressure below (pore pressure above lithostatic), causing hydrofracture and some modification to the flow. Our assumption here is therefore that such tectonically induced changes are sufficiently slow that negative effective pressures do not occur; and in fact, we do not include tectonically induced uplift or subsidence in the model, although it is straightforward to do so. The boundary conditions are thus the flow conditions

$$
\begin{array}{cc}
-\frac{\partial \tilde{p}_{e}}{\partial z}-(1-\phi)=0 & z=0, \\
\tilde{p}_{e}=0 & z=h,
\end{array}
$$

and the kinematic condition applied at the top surface $z=h$, which relates the evolution of $h$ to the rate of sediment accumulation; this is

$$
\dot{h}=v+\left.u^{s}\right|_{z=h},
$$

which can be written in terms of $\tilde{p}_{e}$ in the form

$$
\dot{h}=v(t)+\lambda \bar{k}\left[-\frac{\partial \tilde{p}_{e}}{\partial z}-(1-\phi)\right] \quad z=h
$$

Here $v$ is the dimensionless sedimentation rate which is $O(1)$, through choice of the sedimentation rate scale. Positive values of $v$ correspond to loading and negative values to unloading. 


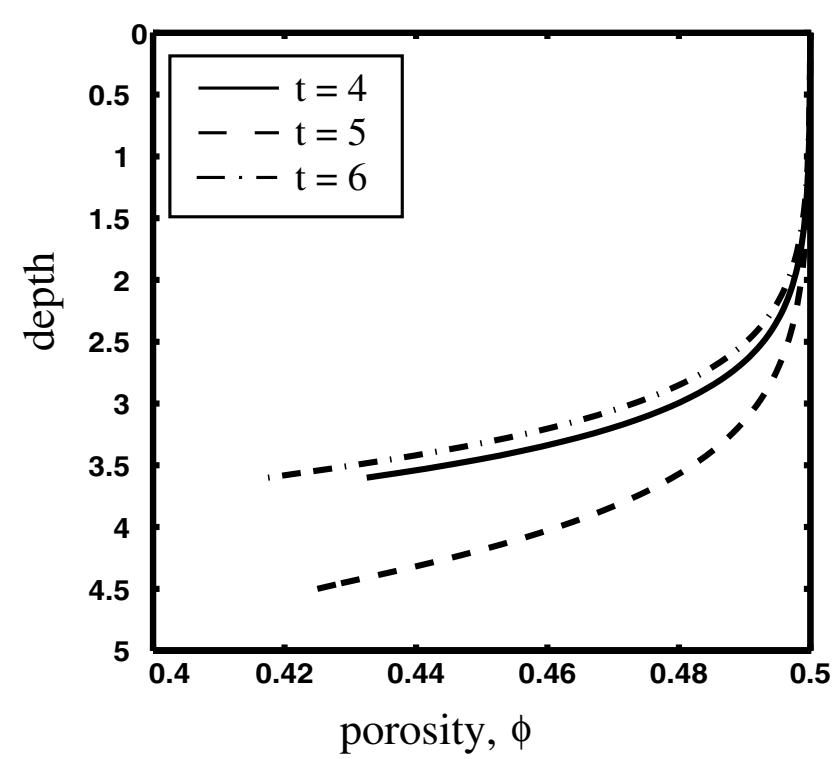

Figure 4. Porosity profile for unloading after an initial loading period in a slowly compacting basin, using values of $\lambda=0.01$ and $\gamma=0.25$. The solutions are shown at times $t=4,5,6$ and indicate the growth of a basal boundary layer. The surface has been loaded with $v=1$ for $0<t<5$ and then unloaded with $v=-1$ for $5<t<6$. The boundary layer proceeds independently of what happens on the basin surface.

[18] In implementing numerical solutions of the model, it is convenient to note that (12) applies when $\tilde{p}_{e}=\tilde{p}_{e}^{*}$ (i.e., on the NCL), and (13) applies when $\tilde{p}_{e}<\tilde{p}_{e}^{*}$ (on the URL): see Figure 3; $\tilde{p}_{e}^{*}$ is the value of $\tilde{p}_{e}$ at the join of the NCL and the current URL. If we are on the URL, then $\tilde{p}_{e}^{*}$ does not change as $\tilde{p}_{e}$ does, and the criterion that we are indeed on the URL is that either $\tilde{p}_{e}<\tilde{p}_{e}^{*}$, or $\tilde{p}_{e}=\tilde{p}_{e}^{*}$ but $\tilde{p}_{e}$ is decreasing. Thus the condition for evolving $p \bullet_{e}^{*}$ on the URL branch is

$$
\begin{gathered}
d \tilde{p}_{e}^{*} / d t_{s}=0 \quad \tilde{p}_{e}=\tilde{p}_{e}^{*} \quad d \tilde{p}_{e} / d t_{s}<0, \\
d \tilde{p}_{e}^{*} / d t_{s}=0 \quad \tilde{p}_{e}<\tilde{p}_{e}^{*} .
\end{gathered}
$$

Alternatively, on the NCL branch, $\tilde{p}_{e}=\tilde{p}_{e}^{*}$ and thus the time derivatives of each are the same, and the criterion to be on the NCL is that $\tilde{p}_{e}$ is increasing. Thus on the NCL branch,

$$
d \tilde{p}_{e}^{*} / d t_{s}=d \tilde{p}_{e} / d t_{s} \quad \tilde{p}_{e}=\tilde{p}_{e}^{*} \quad d \tilde{p}_{e}^{*} / d t_{s}>0
$$

where $d / d t_{s}=\partial / \partial t+u^{s} \partial / \partial z$ is a Lagrangian derivative following the matrix. We will suppose formally that $\left|u^{s}\right| \ll 1$ and thus that strains are relatively small, so that we can approximate $d \tilde{p}_{e}^{*} / d t_{s}$ $\approx \partial \tilde{p}_{e}^{*} \partial t$, which simplifies the numerical method somewhat. This is true for small times, and also if $\lambda$ is small. For the more relevant case where $\lambda$ is large, analysis [Fowler and Yang, 1998] shows that $\left|u^{s}\right|$ increases from its initial value of zero to a limiting value of $\phi_{0}$ at large times when a continual loading is applied. It seems that our assumption is not an exorbitant restriction; furthermore, the incorporation of finite strains actually requires a level of modeling (involving nonlinear viscoelasticity) which is altogether more complex [Fowler and Noon, 1999].

\section{Analysis of the Model}

[19] When continuous loading is applied, the compaction of sediments will follow the NCL, and the analysis in this case is simply that of Audet and Fowler [1992] and Fowler and Yang [1998]. However, when successive loading and unloading occurs, (a)

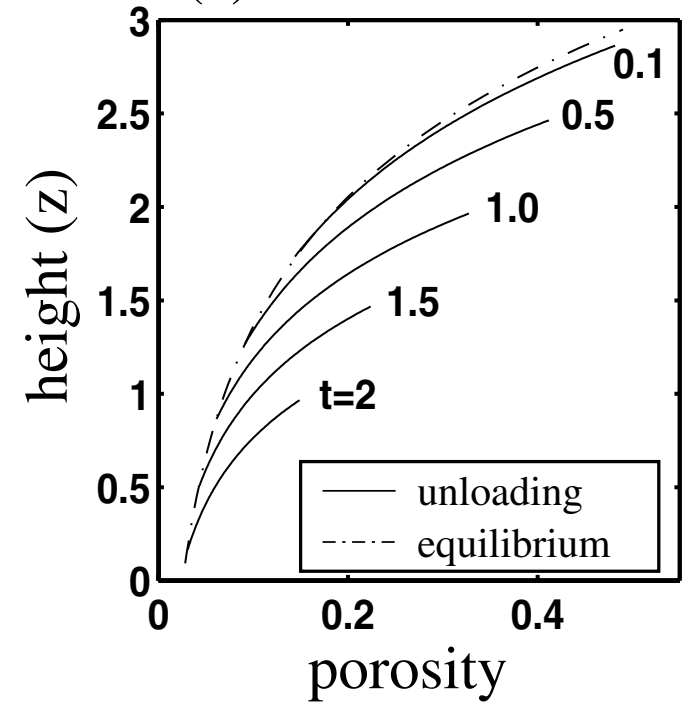

(b)

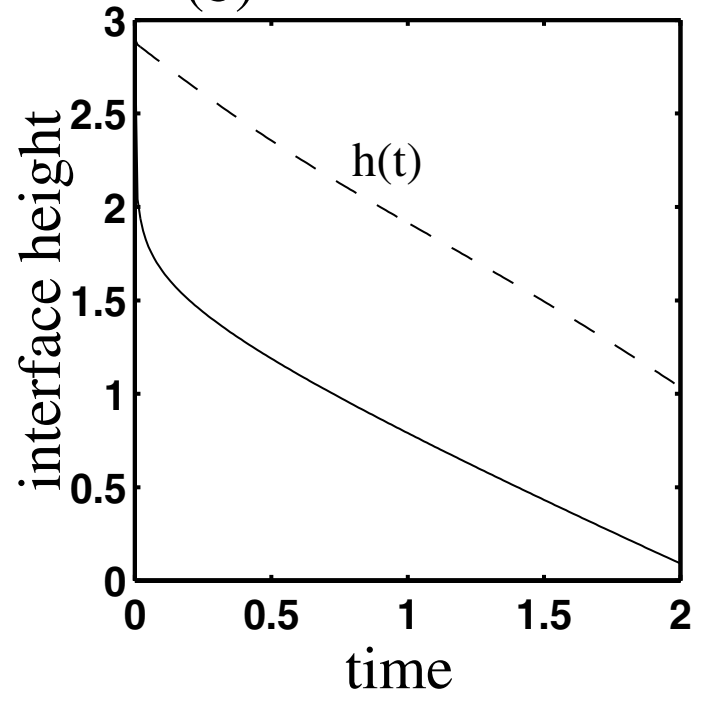

Figure 5. (a) The result of unloading an "equilibrated" Athy exponential porosity profile by imposing $v=-1$. An apparent traveling unloading interface propagates downward at a finite rate. (b) The position of the apparent interface as a function of time. At this interface, there is a small but sharp change in the vertical porosity gradient through a thin transition region which is barely visible. Parameter values are $\lambda=1500$ and $\gamma=0.25$. The rapid initial plunge of the unloading interface can be understood analytically [Yang, 1997], as an approximate expression for the interface depth at small times can be shown to be $(m-1)^{-1} \ln \left\{1+\left[\lambda(m-1)^{2}\left(1-\phi_{0}\right)^{2} t / \gamma \phi_{0}\right]\right\}$. Figure $5 \mathrm{~b}$ shows that the depth of the interface below the surface is approximately constant thereafter. Note that the vertical coordinate plots height above basement, not depth from surface. 


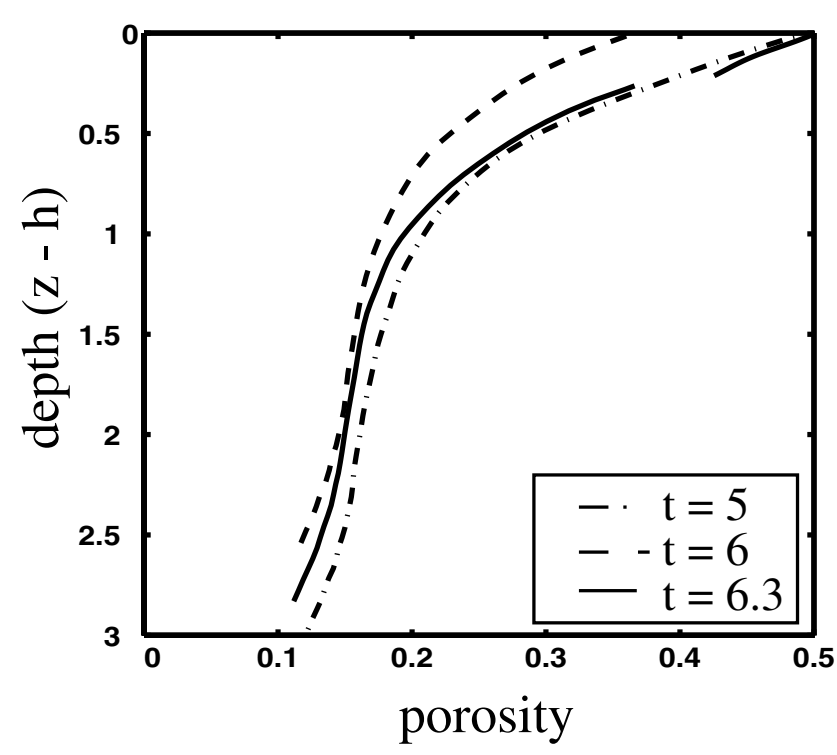

Figure 6. Porosity profiles during cyclic loading and unloading in fast compacting basins $(\lambda=100, \gamma=0.2)$. Loading with $v=1$ occurs for $0<t<5$, then unloading with $v=-1$ for $5<t<6$, and then loading with $v=1$ for $t>6$. Note that the accumulated thickness of sediments at $t=5$ is only $h=3$ because of compaction. The three curves which are shown represent three snapshots: at the end of initial loading $t=5$ (dashed/dotted); the end of unloading $t=$ 6 (dashed); and during reloading at $t=6.3$ (solid). The unloading interface discussed in connection with Figure 5 lies at a height 0.7 above the basement on the reloading curve. It has a slight discontinuity in slope which is not visible to casual inspection. The loading interface on the reloading curve has a discontinuous porosity $\phi$, with a jump from $\sim 0.43$ to $\sim 0.37$ at a depth of 0.2 . Longer unloading periods lead to more dramatic discontinuities.

a succession of interfaces will propagate downward, separating NCL regions from URL regions, and these interfaces separate regions in which the model changes (essentially the diffusion coefficient changes). In solving the model numerically the integral forms of conservation law of mass are used in numerical computations at the interfaces, while the smooth solution away from the interfaces is treated with a standard finite difference method. The finite difference implementation needs a special modification near the moving interface. A three-point interpolation formula of Lagrangian type [Crank, 1975] is used with unequal space intervals near the moving interface. This numerical method is robust in tracing the interfaces.

\subsection{Slow Compaction, $\lambda \ll 1$}

[20] We have previously estimated typical values of the compaction parameter $\lambda$ as $\gtrsim 10^{2}$. It would require extremely rapid rates of deposition even to have $\lambda$ as low as one. For completeness, nevertheless, we discuss the limit in which $\lambda$ is small. In this case the sediments accumulate before there is time to expel the pore water, and as a result an almost lithostatic pore pressure results. Mathematically, the limit $\lambda \ll 1$ corresponds to a singular perturbation, and Audet and Fowler [1992] and Fowler and Yang [1998] showed that a basal boundary layer occurs, with $\phi \approx \phi_{0}$ being approximately constant above this. Physically, the pore pressure is near lithostatic except near the base where compaction is occurring and fluid is expelled upward through the layer.

[21] When we generalize the analysis to the irreversible rheology, we find that the solutions are unaffected, and this is verified by numerical simulations, as indicated in Figure 4 . The compaction of the sediments in the basal boundary layer continues due to the weight of the overlying sediments and is unaffected by surface erosion, since the upper porosity is $\sim \phi_{0}$ independently of whether loading or unloading is occurring.

\subsection{Fast Compaction, $\lambda \gg 1$}

[22] The case when $\lambda \gg 1$ is referred to as fast compaction; it is of more geophysical interest because, as we have seen, typical values of $\lambda$ lie in the range $10^{2}$ for shale to $10^{5}$ for sandstone. It is also more complicated to analyze. Most simply, the limit $\lambda \gg 1$ suggests a rapid relaxation to an equilibrium state in which the pore pressure is hydrostatic and, in fact (with the choice (12)), the porosity decreases exponentially with depth: Athy's law. However, this rapid relaxation only applies if $\lambda \tilde{k} \gg 1$, and this inequality becomes invalid (particularly when $m$ in (11) is large, as we suppose it to be) at a depth where $\phi$ reaches a critical value (such that $\lambda \tilde{k}=1$ ), and below this, the porosity evolution is very slow. For longer times, the exponential profile will extend to greater depth. In order to illustrate the effect on this structure of loading and unloading cycles we consider two particular cases, which are indicative of the new phenomena which the hysteretic model introduces.

3.2.1. Constant unloading from the equilibrium state. [23] The (quasi-)equilibrium state of the nonlinear diffusion equation was given by Fowler and Yang [1998] as the Athy-type solution

$$
\phi^{e}=\phi_{0} e^{-\eta}
$$

where $\eta=h-z$ is the depth below the surface. In Figure 5a we show the result of a numerical experiment in which a previously "equilibrated" porosity profile is unloaded. That is to say, the sedimentary basin is grown for a long time so that the Athy profile is taken up over the whole column. The column is then unloaded by putting $v=-1$ in (17). The numerical results of the unloading response are shown in Figure 5. There is an apparent interface between disturbed and undisturbed regions, which travels rapidly downward initially, but whose speed decreases as it progresses. This interface does not carry a jump in porosity, and in practice, it is unlikely that we could distinguish transient unloading regimes from an equilibrium profile.

3.2.2. Cyclic loading and unloading. [24] The second experiment we choose to illustrate the peculiar properties of the hysteretic rheology is a cyclic loading/unloading experiment. Figure 6 shows a snapshot of a typical simulation. When the surface switches from loading to unloading, there is a rapid propagation downward of the new regime, for the reasons outlined previously, roughly to the base of the equilibrium compaction region. However, below this boundary, the sediments are still compacting, and as a result, there is a mild discontinuity in the porosity gradient because of the jump in diffusion coefficient. This can be seen in Figure 6.

[25] More dramatically, when loading recommences, we find a discontinuity at the loading/unloading surface. At first sight, this appears extraordinary in a diffusive model, but the physical reason is clear. As unloading proceeds, the compacted layers relax along the URL, and thus those buried at depth will never regain the pristine porosity $\phi_{0}$. Hence when reloading occurs, the surface porosity is below $\phi_{0}$, but the newly added material does have porosity $\phi_{0}$ : hence the discontinuity that we see. At the interface the material above moves down the NCL as it is buried, while that below moves down the URL. As burial continues, the discontinuity is diminished and disappears when the material below rejoins the NCL.

[26] So long as the discontinuity is present, the interface moves downward with the solid velocity (since it is a material interface). After it is removed, there remains a (mild) discontinuity in the porosity gradient similar to that at the lower URL/NCL boundary, and this continues to propagate downward. It seems unlikely that 


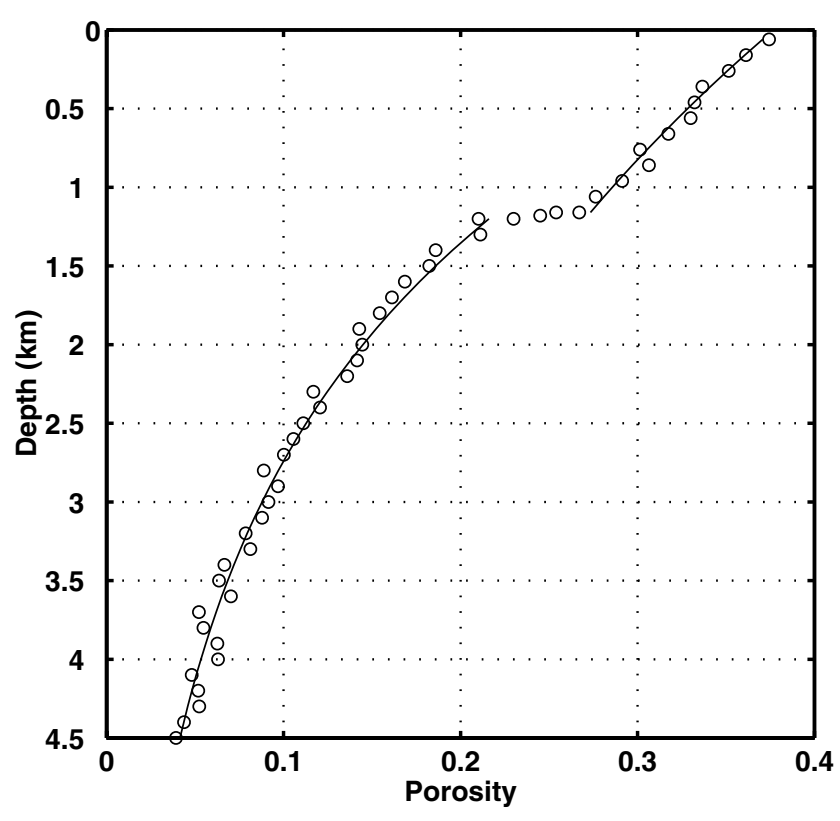

Figure 7. Porosity versus depth in borehole Log N-046 of the China Petroleum and Mineral Exploration Company in the North China Basin. The data are taken at intervals of $1 \mathrm{~m}$ and averaged over distances of $50 \mathrm{~m}$. A clear jump in porosity is seen at a depth of $1200 \mathrm{~m}$. The data were supplied by Q. S. Wang of the Chinese Academy of Sciences. The curves show a theoretical fit to the data using the present model, together with parameter values as described in the text.

such porosity gradient discontinuities could be properly resolved in nature, given the noisiness of the data.

[27] For reasons similar to those in the previous experiment the porosity profile within the top reloading region relaxes rapidly toward the equilibrium Athy profile, and this, together with the approximate unloading profile, gives a potential means for approximately calculating porosity profiles for an arbitrary loading/ unloading sequence, hence (in principle) solving the inverse problem, that of determining sedimentation history from a measured porosity/pore pressure profile.

\section{Conclusions}

[28] We have investigated the nonlinear behavior of sediments during surface loading and unloading in compacting sedimentary basins. The effect of hysteresis in the rheological constitutive law is formulated in terms of a two-branched constitutive law which is consistent with Cam-Clay models and soil experiments. This leads to a model for porosity evolution which takes the form of a Richards-style nonlinear diffusion equation, with a discontinuous diffusion coefficient. The one-dimensional form of the resulting model is controlled by two dimensionless parameters: $\lambda$, which is the ratio of the hydraulic conductivity to the sedimentation rate, and $\gamma$, which is the slope ratio of the decompaction (URL) line to the normal consolidation (NCL) line.

[29] Analytic and numerical results show that the effect of the response to surface unloading is essentially ignorable in the case of slow compaction $(\lambda \ll 1)$ due to the fact that compaction only proceeds within a boundary layer near the basement. Geologically speaking, when the sediment layer is less permeable, or the sediments are deposited rapidly, the system compacts slowly and does not have enough time to respond to changes in surface conditions (whether there be loading or unloading). On the other hand, the response to surface unloading is more complicated and interesting in the case of fast compaction $(\lambda \gg 1)$, which is the usual case. An apparent traveling interface propagates downward when surface unloading begins from a fully compacted equilibrium state where the pore pressure is hydrostatic. The velocity of this interface is independent of the initial state or the surface unloading rate. More generally, a series of interfaces will be generated under conditions of cyclic surface loading and unloading; for a decompacting (URL) region above a compacting (NCL) region, there is a jump in porosity gradient at the interface, whereas for a compacting (NCL) region above a decompacting (URL) region, there is a transiently discontinuous porosity which is gradually reduced, and thereafter a similar porosity gradient to the previous case propagates downward. Furthermore, the porosity profile in such an upper NCL region is nearly in the equilibrium state.

[30] Figure 7 shows a profile of porosity versus depth from a borehole $\log (\log$ N-046) in the North China Basin, obtained using (mainly) sonic velocity and electrical resistivity [Liu and Zhang, 1992]. The tectonic setting is described by Wang and Yang [1997]. The total depth is $4550 \mathrm{~m}$ from the land surface, and the rocks are of mixed type, comprising units of shale and sandstone and, below the jump, limestone. The data were sampled at $\sim 1-\mathrm{m}$ intervals and are quite random, so the circles in Figure 7 represent the data averaged over $50-\mathrm{m}$ intervals. We see a sharp change at a depth of $1150 \mathrm{~m}$ with a porosity jump approximately from 0.27 to 0.22 .

[31] The basin underwent marine sedimentation for $\sim 15 \mathrm{Myr}$, then uplift to the surface (and thus erosion) for perhaps several million years, and then subaerial deposition from the Neogene to the present. The jump in the log data suggests a discontinuous compaction-decompaction interface, and although it could also be associated with unconformity, it may be a consequence of rheological hysteresis.

[32] We have fitted the curves above and below the jump using our model. We choose values $d=1 \mathrm{~km}$ and $\lambda=v_{H} / v_{S}=100$; this implies a relation between sediment type and deposition rate. For example, we might take $v_{H}=3 \times 10^{4} \mathrm{~m} \mathrm{Myr}^{-1}$ for shale, and $v_{S}=$ $300 \mathrm{~m} \mathrm{Myr}^{-1}$. This would imply a timescale of $d / v_{S}=3 \mathrm{Myr}$, and we adopt these scales for illustration. We also choose $m=8, \gamma=$ 0.25 and $\phi_{0}=0.377$.

[33] We load the basin from dimensionless time $t=0$ to $t=t_{L}$, unload until $t=t_{U}$, and then reload from $t=t_{U}$ to the present $t=t_{P}$. Our fitted values are then obtained with $t_{L}=5, t_{U}=7$, and $t_{P}=8.7$, and with dimensionless sedimentation rates of $v=0.9$ below the jump (marine sedimentation), $v=2.4$ above the jump (continental sedimentation), and $v=-0.32$ during unloading. It is clear that the data can be very well fit within the context of our model, although there is no unique choice of parameters to do this.

[34] The present model is clearly not designed to be comprehensive and instead forms part of a modeling strategy to try to separately understand different facets of compaction, such as diagenesis, pressure solution, and so on. Nevertheless, it is worth remarking on some of the more basic assumptions and shortcomings of the model.

[35] The model is one-dimensional. The basic justification for this lies in the large aspect ratio of basins. This allows the use of an approximate one-dimensional model, but as pointed out by Magara [1976a], the widely differing permeabilities of sandstone and shale confounds this assumption in layered systems. Despite this, most theoretical models are one-dimensional.

[36] A different effect of vertically varying lithology within a one-dimensional model is that discontinuous permeabilities and also discontinuous pressure-porosity relations will allow porosity jumps at unconformities. We are unable to offer any useful advice on porosity jumps at unconformities.

[37] There are other effects which may mask porosity discontinuities. For example, the supposed exhumation in the Tertiary in the North Sea is masked in its effect on porosity [Sclater and Christie, 1980] by overpressuring, which could be due to seal formation [Hunt, 1990], diagenesis of dewatering clays, or hydro- 
carbon formation [Bredehoeft et al., 1994]. In short, this paper offers only an analysis of only one of several mechanisms which can occur in the burial and compaction of sediments. However, we believe that the work presented here represents the first attempt to analyze the effect of rheological hysteresis in basin subsidence.

[38] Acknowledgments. We are grateful to Tony Watts of Oxford University for advice and to Q. S. Wang of the Chinese Academy of Sciences for providing the data from the North China Basin.

\section{References}

Allen, P. A., and J. R. Allen, Basin Analysis, Blackwell Sci., Malden, Mass., 1990.

Athy, L. F., Density, porosity, and compaction of sedimentary rocks, $A A P G$ Bull., 14, 1-22, 1930.

Audet, D. M., and A. C. Fowler, A mathematical model for compaction in sedimentary basins, Geophys. J. Int., 110, 577-590, 1992.

Birchwood, R. A., and D. L. Turcotte, A unified approach to geopressuring, low-permeability zone formation, and secondary porosity generation in sedimentary basins, J. Geophys. Res., 99, 20,051-20,058, 1994.

Bredehoeft, J. D., J. B. Wesley, and T. D. Fouch, Simulations of the origins of fluid pressure, fracture generation, and the movement of fluids in the Uinta Basin, Utah, AAPG Bull., 78, 1729-1747, 1994.

Bowles, J. E., Physical and Geotechnical Properties of Soils, McGraw-Hill, New York, 1984.

Burland, J. B., On the compressibility and shear strength of natural clays, Geotechnique, 40, 329-378, 1990.

Cercone, K. R., Thermal history of Michigan Basin, AAPG Bull., 68, 130136, 1984.

Chen, W. F., and E. Mizuno, Nonlinear Analysis in Soil Mechanics: Theory and Implementation, 661 pp., Elsevier Sci., New York, 1990.

Clift, P. D., and J. Turner, Paleogene igneous underplating and subsidence anomalies in the Rockall-Faeroe-Shetland area, Mar. Pet. Geol., 15, 223 244, 1998.

Crank, J., The Mathematics of Diffusion, 2nd ed., Oxford Univ. Press, New York, 1975.

Das, B. M., Advanced Soil Mechanics, McGraw-Hill, New York, 1983.

Dickinson, G., Geologic aspects of abnormal reservoir pressures in Gulf Coast Louisiana, AAPG Bull., 37, 410-432, 1953.

Fowler, A. C., and C. G. Noon, Mathematical models of compaction, consolidation and regional groundwater flow, Geophys. J. Int., 136, $251-260,1999$

Fowler, A. C., and X. S. Yang, Fast and slow compaction in sedimentary basins, SIAM J. Appl. Math., 59, 365-385, 1998.

Fowler, A. C., and X. S. Yang, Pressure solution and viscous compaction in sedimentary basins, J. Geophys. Res., 104, 12,989-12,997, 1999.

Freeze, R. A., and J. A. Cherry, Groundwater, Prentice-Hall, Old Tappan, N. J., 1979.

Gerlach, J. B., and K. R. Cercone, Former Carboniferous overburden in the northern Appalachian Basin-A reconstruction based on vitrinite reflectance, Org. Geochem., 20, 223-232, 1993.

Gibson, R. E., The progress of consolidation in a clay layer increasing in thickness with time, Geotechnique, 8, 171-182, 1958.

Haxby, W. F., and D. L. Turcotte, Stresses induced by the addition or removal of overburden and associated thermal effects, Geology, 4, $181-184,1976$.
Hedberg, H. D., Gravitational compaction of clays and shales, Am. J. Sci., 31, 241-287, 1936.

Hillis, R. R., Regional Tertiary exhumation in and around the United Kingdom, in Basin Inversion, edited by J. G. Buchanan and P. G. Buchanan, Geol. Soc. Spec. Publ., 88, 167-190, 1995.

Hunt, J. M., Generation and migration of petroleum from abnormally pressured fluid compartments, AAPG Bull., 74, 1-12, 1990.

Issler, D. R., A new approach to shale compaction and stratigraphic restoration, Beaufort-MacKenzie Basin and MacKenzie Corridor, northern Canada, AAPG Bull., 76, 1170-1189, 1992.

Jones, M., Mechanical principles of sediment deformation, in The Geological Deformation of Sediments, edited by A. Maltman, pp. 37-71, Chapman and Hall, New York, 1994.

Lewis, C. L. E. P., F. Green, A. Carter, and A. J. Hurford, Elevated K/T palaeotemperatures throughout northwest England: Three kilometres of tertiary erosion?, Earth Planet. Sci. Lett., 112, 131-145, 1992.

Lewis, R. W., and B. A. Schrefler, The Finite Element Method in the Deformation and Consolidation of Porous Media, John Wiley, New York, 1987.

Liu, Y. C., and Y. Q. Zhang, Petroleum geology of China Basins, Pet. Sci., Beijing, 1992

Magara, K., Water expulsion from clastic sediments during compactionDirections and volumes, AAPG Bull., 60, 543-553, $1976 \mathrm{a}$.

Magara, K., Thickness of removed sedimentary rocks, paleopore pressure, and paleotemperature, southwestern part of western Canada Basin, $A A P G$ Bull., 60, 554-565, $1976 \mathrm{~b}$.

Rieke, H. H., and C. V. Chilingarian, Compaction of Argillaceous Sediments, Elsevier Sci., New York, 1974.

Roscoe, K. H., and J. B. Burland, On the generalized stress-strain behavior of wet clay, in Engineering Plasticity, edited by J. Heyman and F. A. Leckie, pp. 535-609, Cambridge Univ. Press, New York, 1968.

Schofield, A. N., and C. P. Wroth, Critical State Soil Mechanics, McGrawHill, New York, 1986.

Sclater, J. G., and P. A. F. Christie, Continental stretching: An explanation of the post Mid-Cretaceous subsidence of the central North Sea basin, J. Geophys. Res., 85, 3711-3739, 1980.

Shannon, P. M., and D. Naylor, Petroleum Basin Studies, Graham and Trotman, Norwell, Mass., 1989.

Smith, J. E., The dynamics of shale compaction and evolution in pore-fluid pressures, Math. Geol., 3, 239-263, 1971.

Wang, Q. S., Characteristics of geophysical field and the outline of deep crustal structure north China region, Acta Geophys. Sin., 35, 319-330, 1992.

Wang, Q. S., and X. S. Yang, Stratified gravity image and its application in Chinese continent, Chin. J. Geophys., 40, 649-659, 1997.

Wangen, M., Pressure and temperature evolution in sedimentary basins, Geophys. J. Int., 110, 601-613, 1992.

Wood, D. M., Soil Behaviour and Critical State Soil Mechanics, Cambridge Univ. Press, New York, 1990.

Yang, X. S., Mathematical modelling of compaction and diagenesis in sedimentary basins, Ph.D. thesis, Univ. of Oxford, Oxford, England, 1997.

A. C. Fowler, Mathematical Institute, University of Oxford, 24-29 St Giles', Oxford OX1 3LB, UK. (Fowler@maths.ox.ac.uk)

X.-S. Yang, Department of Applied Mathematics, University of Leeds, Leeds LS2 9JT, UK. 